\title{
Do Bilateral Pleural Effusions Always Have the Same Cause?
}

\author{
Guillermo Ropero-Luis ${ }^{1}$, Francisco Páez-Codeso ${ }^{2}$, Ricardo Gómez-Huelgas ${ }^{1}$ \\ ${ }^{1}$ Department of Internal Medicine, Hospital Regional Universitario de Málaga, Málaga, Spain \\ ${ }^{2}$ Department of Respiratory Medicine, Hospital Regional Universitario de Málaga, Málaga, Spain
}

Received: 26/02/2019

Accepted: 28/04/2019

Published: $22 / 05 / 2019$

How to cite this article: Ropero-Luis G, Páez-Codeso F, Gómez-Huelgas R. Do bilateral effusions always have the same cause? EJCRIM 2019;6: doi:10.12890/2019_001074.

Conflicts of Interests: The Authors declare that there are no competing interest

This article is licensed under a Commons Attribution Non-Commercial 4.0 License

\section{ABSTRACT}

A 67-year-old man with a history of seropositive rheumatoid arthritis (RA) was admitted to the Internal Medicine ward for bilateral pleural effusion. Two years before this episode, coinciding with an exacerbation of the RA, he was incidentally diagnosed with asymptomatic left pleural effusion compatible with rheumatoid exudate, which was resolved with a tube thoracostomy. Three weeks before admission, the patient developed asthenia, orthopnoea and progressive dyspnoea. A chest x-ray revealed bilateral pleural effusion occupying the lower third of the left hemithorax and a smaller portion of the right hemithorax along with marked elevation of N-terminal fragment of probrain natriuretic peptide levels. The patient was admitted with a diagnosis of left-sided heart failure. Transthoracic echocardiography and cardiac catheterization confirmed the existence of ischaemic cardiomyopathy. After 2 days of diuretic treatment, the right pleural effusion resolved, but the left effusion persisted. A needle thoracentesis was performed, draining $800 \mathrm{ml}$ of milky fluid compatible with rheumatoid pseudochylothorax.

\section{LEARNING POINTS}

- Bilateral pleural effusions nearly always have the same cause, and usually thoracentesis on only one side is needed.

- Rarely, however, there can be two separate causes: this is known as Contarini's syndrome.

\section{KEYWORDS}

Contarini's syndrome, bilateral pleural effusion, rheumatoid arthritis, pseudochylothorax, rheumatoid pleuritis

\section{CASE DESCRIPTION}

A 67-year-old man was admitted to our ward for bilateral pleural effusion (BPE). He had been diagnosed with seropositive rheumatoid arthritis (RA) at the age of 26 and had severe joint deformities. He was being treated with methotrexate 20 mg every week and infliximab $300 \mathrm{mg}$ every 8 weeks. Two years before this episode, coinciding with an exacerbation of the RA, he was incidentally diagnosed with asymptomatic pleural effusion occupying two thirds of the left hemithorax (Fig. 1). The pleural fluid showed features of an exudate compatible with rheumatoid pleuritis (Table 1). The effusion resolved with a tube thoracostomy (2,300 $\mathrm{ml}$ of yellowish fluid was drained) and respiratory physiotherapy (Fig. 2).

Three weeks before admission, the patient developed asthenia, orthopnoea and progressive dyspnoea. He did not report any signs or symptoms suggestive of an exacerbation of the RA. His rheumatologist referred him to the emergency room. Upon admission, the patient was haemodynamically stable and afebrile. His oxygen saturation on ambient air was $90 \%$. He had mild tachypnoea at rest which worsened with conversation. Pulmonary auscultation revealed wet crackles in the lower right hemithorax and diminished vesicular breath sounds with decreased transmission of vocal vibrations in the lower left hemithorax. The rest of the physical examination was unremarkable. 

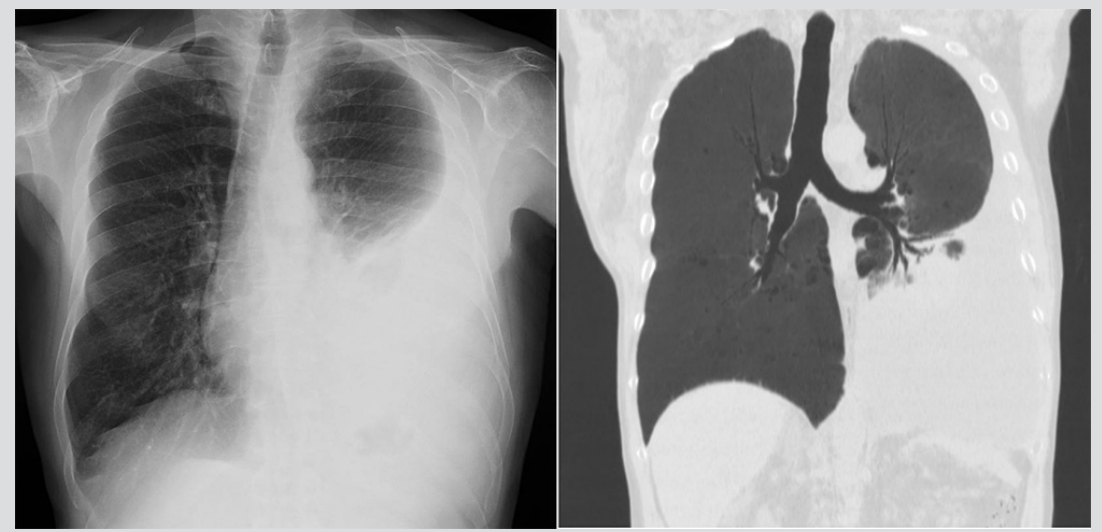

Figure 1. Postero-anterior chest $x$-ray (left) and coronal section of a thoracic CT scan (right) showing pleural effusion occupying two thirds of the left hemithorax

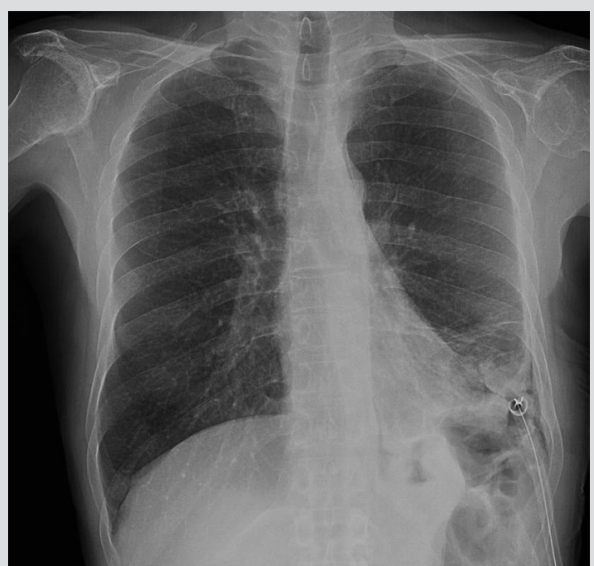

Figure 2. Postero-anterior chest $x$-ray showing a chest tube in the left hemithorax and a significant decrease in the pleural fluid seen in Fig. 1

Blood tests showed moderate elevation of C-reactive protein $(94 \mathrm{mg} / \mathrm{dl}$; normal <2.9), high-sensitivity cardiac troponin I $(0.737 \mathrm{ng} / \mathrm{ml}$; normal $<0.045)$ and $\mathrm{N}$-terminal fragment of pro-brain natriuretic peptide (NT-proBNP) $(5,579 \mathrm{pg} / \mathrm{ml}$; normal <900). An electrocardiogram showed symmetrical inverted T waves in the anterolateral leads with no signs of acute ischaemia. A chest $x$-ray revealed BPE occupying the lower third of the left hemithorax and a smaller portion of the right hemithorax (Fig. 3).

The patient was admitted to the Internal Medicine ward with a diagnosis of left-sided heart failure (HF) and BPE. Depletion therapy with intravenous furosemide was started. A transthoracic echocardiogram showed segmental contractility abnormalities in the left ventricle along with an aneurysmal region in the apex and an ejection fraction on the lower end of normal parameters. These findings were highly suggestive of ischaemic cardiomyopathy. Subsequently, cardiac catheterization was performed which confirmed the presence of diffuse atherosclerosis with moderate lesions in the left anterior descending artery and severe lesions in the circumflex artery (where two drugeluting stents were placed).

After 2 days of diuretic treatment, the patient had improved significantly. He had no dyspnoea or orthopnoea. A chest x-ray showed resolution of the right pleural effusion with persistence of the left effusion (Fig. 4).

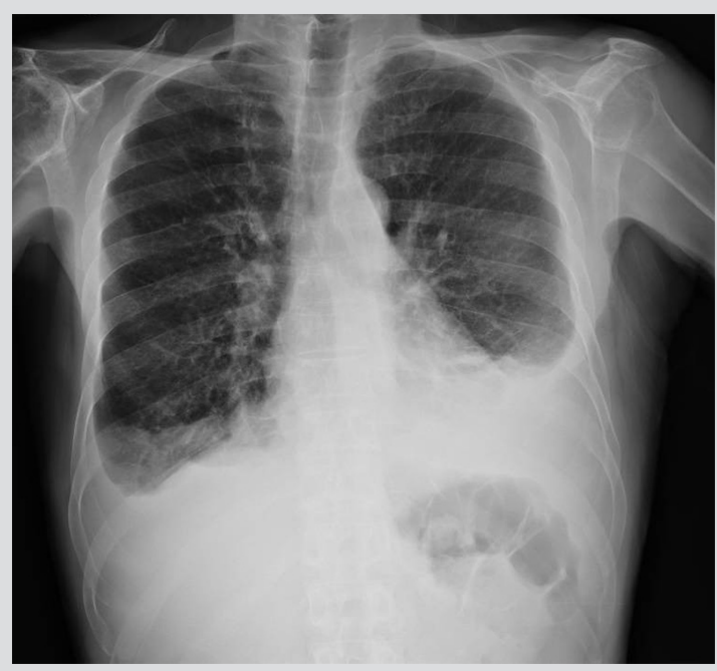

Figure 3. Postero-anterior chest $x$-ray showing bilateral pleural effusion occupying the lower third of the left hemithorax and in a smaller portion of the right hemithorax

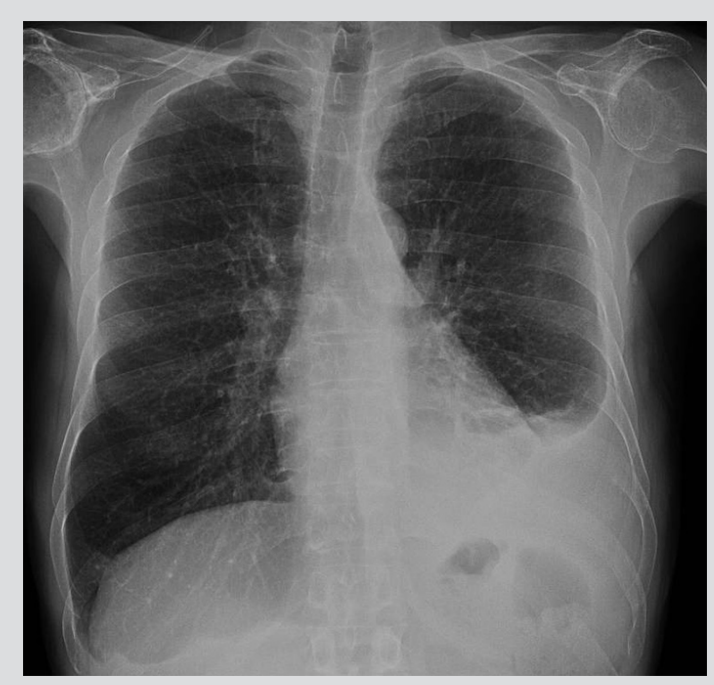

Figure 4. Postero-anterior chest $x$-ray showing resolution of the right pleural effusion and persistence of the left effusion seen in Fig. 3 
As other non-cardiac aetiologies were suspected, a needle thoracentesis was performed, draining 800 ml of milky fluid. Analysis showed features compatible with rheumatoid pseudochylothorax (Table 1). Further tests ruled out tuberculosis. The patient was discharged asymptomatic.

\begin{tabular}{|c|c|c|}
\hline & Exudate & Pseudochylothorax \\
\hline Macroscopic appearance & Yellowish & Milky \\
\hline Leukocytes (per $\mu l)$ & 880 & 600 \\
\hline Neutrophils (\%) & $90 \%$ & $85 \%$ \\
\hline $\mathrm{pH}$ & & 7.28 \\
\hline Glucose (mg/dl) & $<1$ & 64 \\
\hline Protein (g/dl) & 5.3 & 5.3 \\
\hline Lactate dehydrogenase (U/I) & 2,696 & 1,056 \\
\hline Cholesterol (mg/dl) & 91 & 165 \\
\hline Triglycerides (mg/dl) & 17 & 16 \\
\hline Adenosine deaminase (U/I) & 71 & 45 \\
\hline Cytopathology & $\begin{array}{l}\text { Granular background, mesothelial cells } \\
\text { with degenerative changes, mixed } \\
\text { inflammatory exudate, multinucleated } \\
\text { giant cells }\end{array}$ & $\begin{array}{l}\text { Abundant cholesterol crystals, granular } \\
\text { background, mesothelial cells with } \\
\text { reactive changes, acute mixed } \\
\text { inflammatory exudate with neutrophils, } \\
\text { multinucleated giant cells, and foamy } \\
\text { histiocytes }\end{array}$ \\
\hline
\end{tabular}

Table 1. Comparison of the characteristics of the pleural fluid from the first thoracentesis (exudate) and the second (pseudochylothorax)

\section{DISCUSSION}

Contarini's Syndrome

Contarini's syndrome refers to the occurrence of bilateral pleural fluid accumulation with each side due to a different cause ${ }^{[1]}$. Francesco Contarini was the 95th Doge of Venice and died in 1625 . His post-mortem examination revealed that he had right hydrothorax due to HF as well as left empyema ${ }^{[2]}$. It is a very rare condition: its prevalence was $0.9 \%$ in a review of 546 patients with $\mathrm{BPE}^{[1]}$ and $5.6 \%$ in a prospective study of 36 patients ${ }^{[3]}$.

\section{Rheumatoid Pleuritis and Pseudochylothorax}

Rheumatoid pleuritis is an infrequent complication that affects less than 5\% of patients with RA. Features of the pleural fluid usually include an exudate with low $\mathrm{pH}$ and glucose levels and high protein, lactate dehydrogenase and adenosine deaminase levels ${ }^{[4]}$. In 1968 , Nosanchuck and Naylor described the 'rheumatoid cytological triad'[5], considered pathognomonic by some authors: granular background, multinucleated giant cells and fusiform histiocytes. In the context of RA, pseudochylothorax is a rare pleural effusion: RA was the aetiology in $9 \%$ of cases in a review of 174 cases of pseudochylothorax reported up to 1999 (the most frequent aetiology was pleural tuberculosis) ${ }^{[6]}$, and $32 \%$ in a more recent review of 104 cases $^{[7]}$. Its absolute prevalence and pathogenesis are unknown, although it is associated with longterm effusions. Pseudochylothorax appears as a milky or turbid fluid with cholesterol levels usually $>200 \mathrm{mg} / \mathrm{dl}$ and triglyceride levels $<100$ $\mathrm{mg} / \mathrm{dl}^{[8]}$. A cholesterol/triglyceride ratio $>1$ and the presence of cholesterol crystals on cytopathology are the most useful diagnostic tests ${ }^{[7]}$.

\section{Workup of the Patient with BPE}

There are no clinical guidelines on the diagnosis and management of BPE, so we suggest following the diagnostic decision algorithm proposed by Ferreiro et al. ${ }^{[9]}$. If the clinical picture suggests a transudate, treating the cause without initial thoracentesis is warranted. If the clinical picture does not suggest any diagnosis, or the evolution with treatment is not favourable, a unilateral thoracentesis should be performed. The most common cause of BPE is HF, and the measurement of NT-proBNP levels in blood has high diagnostic accuracy in patients with pleural effusion ${ }^{[10]}$, so it can help in deciding whether or not to perform thoracentesis. 
Clinical characteristics suggestive of different aetiologies include ${ }^{[3]}$ : fever or pleuritic pain in a patient with decompensated HF, unilateral involvement of the lung parenchyma, effusions of significantly different sizes or features on imaging tests, and resolution of only one side. Then, if the pleural effusion is determined to be a transudate and the clinical data suggest a systemic known disease, the corresponding treatment should be started without contralateral thoracentesis being performed. If it does not, or the treatment fails, bilateral diagnostic thoracentesis should be performed. Imaging techniques may help select patients who would benefit the most from the procedure ${ }^{[3]}$. Ultrasonography is more sensitive than CT for detecting complications within the effusion, and can be used to assess its characteristics [11]: transudates are anechoic; thickened pleura, parenchymal lesions, complex septated and non-septated or homogeneously echogenic patterns are indicative of exudates. The complication rate of bilateral thoracentesis is comparable to that of the unilateral procedure ${ }^{[12]}$. In our case, the marked clinical improvement and resolution of the right pleural effusion with diuretic therapy confirmed its cardiac origin, leading us to reconsider the aetiology of the left effusion. The pseudochylothorax was probably the result of the chronic progression of the previously diagnosed rheumatoid pleuritis.

\section{REFERENCES}

1. Porcel JM, Civit MC, Bielsa S, Light RW. Contarini's syndrome: bilateral pleural effusion, each side from different causes. J Hosp Med 2012;7(2):164-165.

2. Kutty CP, Varkey B. "Contarini's condition:" bilateral pleural effusion with markedly different characteristics. Chest 1978;74:679-680.

3. Ferreiro L, San José ME, Gude F, Lama A, Suárez-Antelo J, Golpe A, et al. Unilateral or bilateral thoracocentesis for bilateral pleural effusion. A prospective study. Arch Bronconeumol 2016;52(4):189-195.

4. Balbier-Gurman A, Yigla M, Nahir AM, Braun-Moscovici Y. Rheumatoid pleural effusion. Semin Arthritis Rheum 2006;35:368-378.

5. Nosanchuck JS, Naylor B. A unique cytology picture in pleural fluid from patients with rheumatoid pleuritis. Am J Clin Pathol 1968;50:330-335.

6. Garcia-Zamalloa A, Ruiz-Irastorza G, Aguayo FJ, Gurrutxaga N. Pseudochylothorax. Report of 2 cases and review of the literature. Medicine (Baltimore) 1999;78(3):200-207.

7. Lama A, Ferreiro L, Toubes ME, Golpe A, Gude F, Álvarez-Dobaño JM, et al. Characteristics of patients with pseudochylothorax-a systematic review. J Thorac Dis 2016;8:20932101.

8. Páez-Codeso FM, Pérez Soriano MP, Casado Miranda E, Dorado Galindo A, Bautista Ojeda MD. Cytopathology and biochemistry of the rheumatoid pleural fluid. Presentation of a case. Rev Lab Clin 2018 [in press]. doi.org/10.1016/j.labcli.2018.07.001

9. Ferreiro L, San José E, Antelo JS, Valdés L. Bilateral pleural effusion: a proposed diagnostic decision algorithm. Ann Am Thorac Soc 2016;13(10):1865-1867.

10. Han ZJ, Wu XD, Cheng JJ, Zhao SD, Gao MZ, Huang HY, et al. Diagnostic accuracy of natriuretic peptides for heart failure in patients with pleural effusion: a systematic review and updated meta-analysis. PLoS One 2015;10(8):e0134376.

11. Yang PC, Luh KT, Chang DB, Wu HD, Yu CJ, Kuo SH. Value of sonography in determining the nature of pleural effusion: analysis of 320 cases. AJRAm J Roentgenol 1992;159(1):2933.

12. Vollmer I, Benegas M, Sánchez M. Is bilateral thoracocentesis necessary in bilateral pleural effusion? Arch Bronconeumol 2016;52(7):345-346 